University of Nebraska - Lincoln

DigitalCommons@University of Nebraska - Lincoln

June 1996

\title{
Static-electric-field effects on high harmonic generation
}

\author{
Min-Qi Bao \\ Institute for Theoretical Atomic and Molecular Physics, Harvard-Smithsonian Center for Astrophysics, \\ Cambridge, Massachusetts
}

Anthony F. Starace

University of Nebraska-Lincoln, astarace1@unl.edu

Follow this and additional works at: https://digitalcommons.unl.edu/physicsstarace

Part of the Physics Commons

Bao, Min-Qi and Starace, Anthony F., "Static-electric-field effects on high harmonic generation" (1996). Anthony F. Starace Publications. 56.

https://digitalcommons.unl.edu/physicsstarace/56

This Article is brought to you for free and open access by the Research Papers in Physics and Astronomy at DigitalCommons@University of Nebraska - Lincoln. It has been accepted for inclusion in Anthony F. Starace Publications by an authorized administrator of DigitalCommons@University of Nebraska - Lincoln. 


\title{
Static-electric-field effects on high harmonic generation
}

\author{
Min-Qi Bao* and Anthony F. Starace ${ }^{\dagger}$ \\ Institute for Theoretical Atomic and Molecular Physics, Harvard-Smithsonian Center for Astrophysics, 60 Garden Street MS-14, \\ Cambridge, Massachusetts 02138
}

(Received 21 December 1995)

\begin{abstract}
High harmonic generation in the presence of a static electric field is analyzed theoretically by an extension of the zero-range potential model treatment of Becker et al. [Phys. Rev. A 50, 1540 (1994)]. For $\mathrm{H}^{-}$and a $\mathrm{CO}_{2}$ laser of $5 \times 10^{10} \mathrm{~W} / \mathrm{cm}^{2}$ intensity, a static electric field of $100 \mathrm{kV} / \mathrm{cm}$ is shown to produce a plateau from the 3rd to the 23rd harmonic in which neighboring even and odd harmonics are of comparable intensity. Furthermore, odd harmonics on the plateau are one or more orders of magnitude more intense than in the absence of the static electric field. Dependence of emission rates on laser intensity and results for harmonic generation by Ar are also presented. [S1050-2947(96)50106-X]
\end{abstract}

PACS number(s): 32.80.Wr, 42.65.Ky, 42.50.Hz, 32.80.Qk

Experimental observation [1] that an atomic gas exposed to intense laser radiation can generate harmonics having comparable intensity over a "plateau" region of a few dozen harmonic orders stimulated much experimental and theoretical effort to understand the phenomenon, as has been reviewed elsewhere [2-6]. Recently harmonics of order 109 [7] and 135 [8] have been observed in rare gases for laser intensities of $10^{15} \mathrm{~W} / \mathrm{cm}^{2}$. Generation of such high-order harmonics with significant intensity may lead to useful sources of short-pulse, short-wavelength coherent radiation [5]. Key features of the experimental observations are now well understood [9] (or at least reproducible theoretically), and appear to be general features of strongly driven harmonic systems. In particular, Becker et al. [10] and others $[7,11]$ have shown that a zero-range potential model provides a good description of the observed phenomena.

In this paper we present single-atom or -ion theoretical results on high harmonic generation in the presence of a static electric field using an extension of the zero-range potential model treatment of Becker et al. [10]. Results are presented for two cases treated in Ref. [10]: harmonic generation by $\mathrm{H}^{-}$using a $\mathrm{CO}_{2}$ laser and by $\mathrm{Ar}$ using a neodymium-doped yttrium aluminum garnet (Nd:YAG) laser. For a static field of $100 \mathrm{kV} / \mathrm{cm}$ we find strong production of even harmonics. Similar intense production of high even harmonics has been seen by Perry and Crane [12] in a twocolor experiment. Furthermore we find that the intensities of odd harmonics near the low-order end of the plateau are increased by orders of magnitude. Such large increases in odd high harmonics on the plateau, particularly the low orders, have been predicted theoretically recently for two-color high harmonic generation when the frequencies are multiples of one another and when the two fields have comparable intensities $[13,14]$. We find increases in the harmonic emission rates of similar magnitude using only a single laser fre-

\footnotetext{
*Permanent address: Quantum Development Corporation, 2700 Philadelphia Pike, Claymont, DE 19703.

†Permanent address: Department of Physics and Astronomy, The University of Nebraska-Lincoln, 116 Brace Laboratory, Lincoln, NE 68588-0111.
}

quency and a static electric field whose amplitude is less than $2 \%$ of that of the laser field. A static field thus appears to be a sensitive means for controlling the generation of both even and odd high harmonics.

As Becker et al. [10] have presented the zero-range potential model for high harmonic generation in complete detail, we restrict ourselves here to introducing the changes in the formulation and in the results due to the static electric field. Details of our calculation will be presented elsewhere [15]. We approximate the potential of the atom or ion by the zero-range potential [16-18]

$$
V(r)=\frac{2 \pi}{\kappa} \delta(\mathbf{r}) \frac{\partial}{\partial r} r
$$

where $\kappa=\sqrt{2\left|E_{b}\right|}$ and $E_{b}$ is the energy of the bound state. The laser field and the static field are described by

$$
\mathbf{E}(t)=\left(E_{S}+E_{L} \sin \omega t\right) \hat{\mathbf{k}}
$$

and the vector potential is

$$
\mathbf{A}(t)=c\left(\frac{E_{L}}{\omega} \cos \omega t-E_{S} t\right) \hat{\mathbf{k}},
$$

where $c$ is the velocity of light, $\omega$ is the laser frequency, and $E_{S}$ and $E_{L}$ are the amplitudes of the static and laser fields.

The solution of the time-dependent Schrödinger equation,

$$
i \frac{\partial}{\partial t} \psi(\mathbf{r}, t)=\left[-\frac{1}{2 m} \nabla^{2}+V(r)-e \mathbf{r} \cdot \mathbf{E}(t)\right] \psi(\mathbf{r}, t),
$$

is

$$
\psi(\mathbf{r}, t)=\int d^{3} r^{\prime} \int_{-\infty}^{t} d t^{\prime} G\left(\mathbf{r}, t ; \mathbf{r}^{\prime}, t^{\prime}\right) V\left(r^{\prime}\right) \psi\left(\mathbf{r}^{\prime}, t\right)
$$

The Green's function describing propagation of the electron in both the laser and static electric fields is given by

$$
\begin{aligned}
G\left(\mathbf{r}, t ; \mathbf{r}^{\prime}, t^{\prime}\right)= & G^{(0)}\left(\mathbf{r}-\mathbf{r}^{\prime}, t-t^{\prime}\right) \exp \left[-i R\left(\mathbf{r}, t ; \mathbf{r}^{\prime}, t\right)\right] \\
& \times \exp \left[-i M\left(t, t^{\prime}\right)\right],
\end{aligned}
$$


where

$$
\begin{gathered}
R\left(\mathbf{r}, t ; \mathbf{r}^{\prime}, t^{\prime}\right)=e\left[\mathbf{A}(t) \cdot \mathbf{r}-\mathbf{A}\left(t^{\prime}\right) \cdot \mathbf{r}^{\prime}-\frac{\mathbf{r}-\mathbf{r}^{\prime}}{t-t^{\prime}} \int_{t^{\prime}}^{t} d \tau \mathbf{A}(\tau)\right] \\
M\left(t^{\prime}, t^{\prime \prime}\right)= \\
\frac{e^{2}}{2 m}\left\{\int_{t^{\prime \prime}}^{t^{\prime}} d \tau[\mathbf{A}(\tau)]^{2}\right. \\
\left.-\frac{1}{t^{\prime}-t^{\prime \prime}}\left[\int_{t^{\prime \prime}}^{t^{\prime}} d \tau \mathbf{A}(\tau)\right]^{2}\right\}
\end{gathered}
$$

and

$$
G^{(0)}(\mathbf{r}, t)=\theta(t)\left[\frac{i m}{2 \pi(t-i \boldsymbol{\epsilon})}\right]^{3 / 2} \exp \left(i \frac{m r^{2}}{2 t}\right)
$$

This Green's function may be obtained using a symbolic manipulation code [19].

The form of the atom or ion potential in Eq. (1) greatly simplifies the solution of Eq. (5) since the $\delta$ function in $\mathbf{r}^{\prime}$ collapses the spatial integration $[10,15]$. Hence Eq. (5) reduces to a one-dimensional integral equation, which can be solved by employing a quasienergy representation for $\psi(\mathbf{r}, t)$, i.e.,

$$
\lim _{\mathbf{r} \rightarrow 0} \frac{\partial}{\partial r} r \psi(\mathbf{r}, t)=e^{-i E t} W(t)
$$

Following Becker et al. [10], we set $E$ equal to the energy of the initial state, $E=E_{b}=-\kappa^{2} / 2$, thereby neglecting the small ac Stark shift. (As noted by Becker et al. [10], the ponderomotive shift is retained, since it is included in the Green's function $G$.) Also, we assume that states other than the initial state are not significantly populated, so that $W(t) \equiv \kappa(\kappa / 2 \pi)^{1 / 2}[10]$. These two approximations for $E$ and $W(t)$ in Eq. (10) allow one to obtain an analytic solution for $\psi(\mathbf{r}, t)$ from Eq. (5) that describes the wave function of the electron in all three fields: the potential in Eq. (1) and the two external fields in Eq. (2).

The amplitude for generation of a harmonic photon of frequency $\Omega$ is proportional to the Fourier transform of the dipole moment,

$$
\mathbf{d}(\Omega)=\int d t \int d^{3} \mathbf{r} e^{i \Omega t} \Psi^{*}(\mathbf{r}, t) \hat{\boldsymbol{\epsilon}} \cdot \mathbf{r} \Psi(\mathbf{r}, t)
$$

where $\Psi(\mathbf{r}, t)$ is the solution of Eq. (5) and $\hat{\epsilon}$ is the polarization of the emitted photon. Implicit in Eq. (11) is the dipole approximation. Since $\Psi(\mathbf{r}, t)$ is a dressed state, it is not an eigenstate of parity and hence the amplitude in Eq. (11) is in general nonzero. While harmonic generation rates should in principle be based on the dipole correlation function, use of the dipole expectation value in single-atom theories is common and has been shown to be justified when interatomic correlation in a gas sample may be neglected [20]. Using the wave function $\Psi(\mathbf{r}, t)$ defined by Eqs. (5)(10), Eq. (11) may be reduced (by rather detailed but straightforward algebra [21]) to

$$
\mathbf{d}(\Omega)=-i\left(\frac{\pi}{\omega}\right)^{1 / 2} \sum_{k, k^{\prime}} \delta\left(\Omega-\left(2 k-k^{\prime}+1\right) \omega\right) L_{k, k^{\prime}}^{*} \hat{\mathbf{k}}
$$

where $L_{k, k^{\prime}}^{*}$ is a dimensionless amplitude defined by

$$
\begin{aligned}
L_{k, k^{\prime}}^{*}= & 2\left(\frac{\left|E_{b}\right|}{\omega}\right)^{1 / 2} \eta^{1 / 2} \frac{i^{2 k+k^{\prime}}}{\left(2 k-k^{\prime}+1\right)^{2}} \\
& \times \int_{0}^{\infty} \frac{d \tau}{\tau^{3 / 2}} \exp \left[i\left(k-\frac{k^{\prime}}{2}+\frac{1}{2}\right) \tau\right] \\
& \times\left\{i J_{k^{\prime}}(v)\left[J_{k+1}(z) \beta_{+}(\tau) \sin \alpha-J_{k}(z) \beta_{-}(\tau) \cos \alpha\right]\right. \\
& \left.-J_{k}(z) J_{k^{\prime}-1}(v) R\left(\tau+\frac{i}{k-\frac{k^{\prime}}{2}+\frac{1}{2}}\right) \sin \alpha\right\}
\end{aligned}
$$

which involves only a single integral to be evaluated numerically. Equation (13) involves two field-dependent parameters, $R \equiv E_{S} / E_{L}$ and $\eta \equiv c^{2} E_{L}^{2} /\left(4 \omega^{3}\right)=U_{P} / \omega$, where $U_{P}$ is the ponderomotive potential. The arguments of the Bessel functions and the functions $\alpha$ and $\beta$ in Eq. (13) depend on the parameter $\tau$ [where $\tau \equiv \omega\left(t^{\prime}-t\right)$, and $t$ and $t^{\prime}$ are defined in Eq. (5)]:

$$
\begin{gathered}
z(\tau)=\eta\left[\sin \tau-\frac{4 \sin ^{2}(\tau / 2)}{\tau}\right] \\
v(\tau)=4 \eta R[2 \sin (\tau / 2)-\tau \cos (\tau / 2)] \\
\beta_{ \pm}(\tau)=\frac{\left(2 k+1-k^{\prime}\right)}{2 k+1-k^{\prime} \pm 1} e^{ \pm i|\tau| / 2}-\frac{2}{\tau} \sin \left(\frac{\tau}{2}\right), \\
\alpha(\tau)=\frac{\left|E_{b}\right|}{\omega} \tau+\eta \tau\left[1-\left(\frac{\sin (\tau / 2)}{\tau / 2}\right)^{2}\right]-\frac{\pi}{2}\left(k+k^{\prime}+\frac{1}{2}\right) .
\end{gathered}
$$

In the limit $E_{S} \rightarrow 0$, we have that $R \rightarrow 0$ and $v \rightarrow 0$, so that $J_{k^{\prime}}(v) \rightarrow \delta_{k^{\prime} 0}$. Hence, the static field $E_{S}$ is responsible for the second term in curly brackets in Eq. (13) as well as for the summation over $k^{\prime}$ in Eq. (12). This second summation over Bessel functions $J_{k^{\prime}}(v)$ greatly increases the numerical effort required to obtain harmonic generation rates, since for a given order harmonic $n$ all amplitudes $L_{k, k^{\prime}}^{*}$ having $2 k+1-k^{\prime}=n$ must be calculated and summed coherently, i.e.,

$$
L_{n}^{*}=\sum_{k, k^{\prime}} L_{k, k^{\prime}}^{*} \delta_{n, 2 k+1-k^{\prime}}
$$

This contrasts with the case of a single frequency in the absence of the static field, for which $k^{\prime}=0$ and only the amplitude $L_{k, 0}^{*}$ (where $2 k+1=n$ ) is nonzero. (Similar complexity, of course, attends the case of two-color harmonic generation $[13,14]$.) In the limit $E_{S} \rightarrow 0, L_{n}^{*}$ in Eq. (21) reduces to the amplitude $L_{k}^{*}$ defined by Becker et al. [22], where $n \equiv 2 k+1$. 


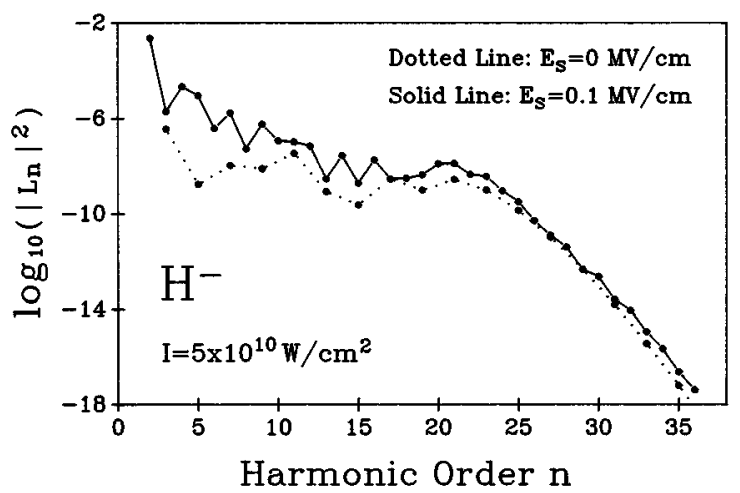

FIG. 1. Absolute squared harmonic generation amplitudes, $\left|L_{n}\right|^{2}$ [cf. Eq. (18)] vs harmonic order $n$ for $\mathrm{H}^{-}$by a $\mathrm{CO}_{2}$ laser having $I=5 \times 10^{10} \mathrm{~W} / \mathrm{cm}^{2}$. Note that $L_{n}$ is dimensionless. Dotted curve connects results for odd harmonics when the static electric field amplitude $E_{S}=0$. Solid curve connects results for even and odd harmonics for $E_{S}=10^{5} \mathrm{~V} / \mathrm{cm}$.

In Fig. 1, we present results for the squared amplitudes $\left|L_{n}\right|^{2}$ for harmonic generation in $\mathrm{H}^{-}$both with and without a static electric field, $E_{S}$. (The harmonic emission rates are proportional to $n^{3}\left|L_{n}\right|^{2}$ [10].) These results are for a $\mathrm{CO}_{2}$ laser of intensity $I=5 \times 10^{10} \mathrm{~W} / \mathrm{cm}^{2}$. The dotted line shows the relative emission rates in the absence of a static electric field; these are identical to those of Becker et al. [10]. In the presence of a static field of amplitude $E_{S}=100 \mathrm{kV} / \mathrm{cm}$, the fifth harmonic emission rate increases by about four orders of magnitude and the seventh and ninth harmonic rates increase by about two orders of magnitude. Furthermore, the even harmonics have rates that are comparable to those for the odd harmonics. These large increases in relative harmonic emission occur for a static electric field amplitude that is only $1.65 \%$ that of the laser (i.e., $R=0.0165$ ).

In contrast, similar calculations for harmonic emission in Ar show less dramatic effects. However, these results, shown in Fig. 2, are for a Nd:YAG laser with intensity $I=3 \times 10^{13}$ $\mathrm{W} / \mathrm{cm}^{2}$. In the absence of the static field, our results again agree with those of Becker et al. [10]. In the presence of a static electric field of amplitude $E_{S}=100 \mathrm{kV} / \mathrm{cm}$, the 5 th through 11th odd harmonics increase in intensity by about one to two orders of magnitude. The even harmonics are 2-4 orders of magnitude less intense than neighboring odd hamonics. Note, however, that the static electric field ampli-

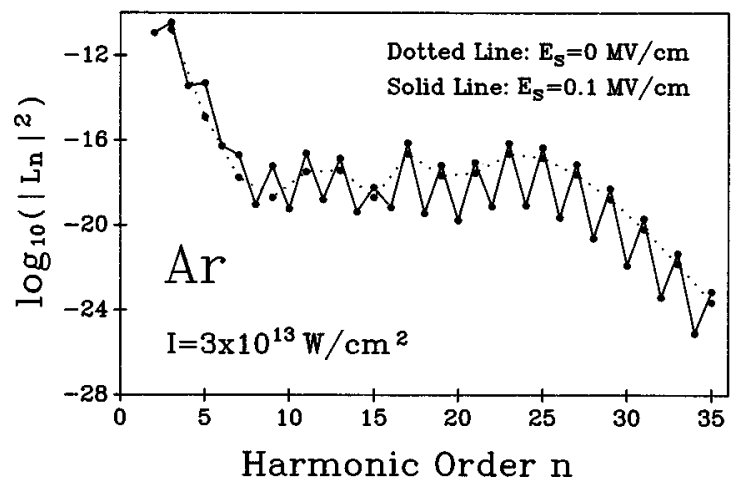

FIG. 2. Same as Fig. 1 for Ar by a Nd:YAG laser having $I=3 \times 10^{13} \mathrm{~W} / \mathrm{cm}^{2}$. tude is only $0.07 \%$ of that of the laser field (i.e., $R=6.7 \times 10^{-4}$ ).

How does one interpret the sensitivity of the relative harmonic generation rates shown in Figs. 1 and 2 to the static electric field? Certainly, the static field polarizes the detached electron wave function, thereby enhancing the magnitude of the dipole amplitude in Eq. (11). According to classical arguments [23-26], harmonic generation requires the active electron to return to the atom or ion at the origin so that it can give up its kinetic energy, producing a harmonic photon. Electron drift away from the origin reduces the rate of harmonic generation. Certainly the static electric field reflects back to the origin some of the electron amplitude traveling in the direction of increasing electrostatic potential energy. (However, determination of the magnitude of the effect of such reflection on the harmonic generation rate would require using a short laser pulse and doing a wave-packet calculation [27], which we have not done.) It is significant in this regard that the most dramatic effects are at the low-order end of the harmonic plateau, as classically the electrons having the lowest kinetic energies are those that are reflected first. (The effect of $E_{S}$ on the high harmonic cutoff $[25,26]$ is to extend it by one harmonic in our $\mathrm{H}^{-}$case and not at all in our Ar case.) It is also interesting that the strongest effect of the static electric field occurs in the tunneling regime; i.e., the Keldysh parameter [28], $\gamma \equiv\left(\left|E_{b}\right| / 2 U_{P}\right)^{1 / 2}$, is 0.85 for our $\mathrm{H}^{-}$calculation but 1.6 for our Ar calculation. (For $\gamma<1$, the laser field lowers the atomic binding potential so that the electron may tunnel out.) However, our two calculations also have very different ratios $R$ of the static to laser field strengths. The effects of both parameters, $\gamma$ and $R$, clearly need to be explored over a large parameter space [15], although this will be quite computationally demanding.

Recently, theoretical predictions for two-color high harmonic generation $[13,14]$ have found similar increases in the harmonic generation rates in cases in which the two frequencies are commensurate. However, each of the two lasers was of comparable intensity. The increase in the harmonic generation rate was interpreted as due to the second laser's keeping the electron close to the nucleus. A two-color experiment [12] (in which the frequency $\omega$ had an amplitude $4.5 \%$ that of the frequency $2 \omega$ ) observed production of even $2 \omega$ harmonics comparable in intensity to the odd $2 \omega$ harmonics. However, the odd harmonics generally decreased in intensity when the weak field was turned on. Superficially, our results might be interpreted as a limiting case of a two-color calculation. However, our static field amplitude in the two cases we treat is two to four orders of magnitude smaller than that of the laser field amplitude and the effect on the harmonic generation rate is as large or larger than that found in either the two-color calculations $[13,14]$ or the two-color experiment [12].

Examining the numerics of our evaluation of Eq. (13), there is not any overriding explanation for the sensitivity of our results to the static electric field. We have verified that for particular harmonics on the plateau, the harmonic generation rates increase smoothly with increasing values of $E_{S}$. The largest partial amplitudes $L_{k, k^{\prime}}$ contributing to the total amplitude $L_{n}$ for the $n$th odd harmonic [cf. Eq. (18)] are $\left(k, k^{\prime}\right)=(k, 0), \quad(k-1,-2), \quad$ and $(k+1,2)$, where $n=2 k+1-k^{\prime}$. However, to be certain that our results have 
converged, we have calculated up to 200 amplitudes for any particular harmonic. Each of these amplitudes involves an integral over $\tau$ whose integrand is strongly oscillatory. On the plateau, the lowest harmonics have amplitudes that are approximately converged by $\tau=50$ a.u.; the highest harmonics are approximately converged by $\tau=20$ a.u. However, in all cases we have carried out the integration to $\tau=200$ a.u.

Competition between harmonic generation and multiphoton ionization has long been of interest as another probe of the final-state dynamics [29-31]. Becker et al. [31] have found that, as a function of laser intensity, the harmonic emission rates for odd harmonics have cusplike structures at intensities corresponding to the closing of an above-threshold-ionization (ATI) threshold by the ponderomotive potential, that is, near integer values of $\left(\left|E_{b}\right|+U_{P}\right) / \omega$. In fact, the cusps are sharp near the eveninteger thresholds, and smoother near odd-integer thresholds. We have calculated the fourth and seventh harmonics of $\mathrm{H}^{-}$as a function of $\mathrm{CO}_{2}$ laser intensity in the presence of a static electric field [15]. We find narrow peaks in the fourth harmonic emission rate at intensities corresponding to integer values of $\left(\left|E_{b}\right|+U_{P}\right) / \omega$, i.e., at the above-thresholddetachment (ATD) thresholds. The seventh harmonic rate, calculated for a frequency-tripled $\mathrm{CO}_{2}$ laser, is similar in shape but larger in magnitude than that of Ref. [31]. However, we find no cusplike structures in either case in the presence of the static field.

In summary, we have used a well-established, short-range potential model treatment [10] to describe the generation of high harmonics in the presence of a static electric field. We find that the harmonic generation rates for odd harmonics at the low end of the plateau increase by orders of magnitude owing to the static electric field, even though the static field amplitude is two to four orders of magnitude smaller than the laser field amplitudes. In addition, significant production of even harmonics is predicted. While the sensitivity of the harmonic generation rates to the static electric field is not completely understood, our results are consistent with both experimental [12] and theoretical $[13,14]$ results for two-color high harmonic generation, although the static field appears to have a stronger effect. Thus, a static electric field appears to be a very sensitive means for controlling high harmonic emission rates and, in particular, for producing high even harmonics.

We thank W. Becker, M. Gavrila, A. L'Huillier, K. C. Kulander, S. Long, K. J. Schafer, and R. Shakeshaft for helpful discussions. This work was supported in part by NSF Grant No. PHY-9410850.
[1] M. Ferray, A. L'Huillier, X. F. Li, L.-A. Lompré, G. Mainfray, and C. Manus, J. Phys. B 21, L31 (1988).

[2] J. H. Eberly, J. Javanainen, and K. Rzazewski, Phys. Rep. 204, 332 (1991)

[3] G. Mainfray and C. Manus, Rep. Prog. Phys. 54, 1333 (1991).

[4] A. L'Huillier, K. J. Schafer, and K. C. Kulander, J. Phys. B 24, 3315 (1991).

[5] A. L'Huillier, L.-A. Lompré, G. Mainfray, and C. Manus, in Atoms in Intense Fields, edited by M. Gavrila (Academic, New York, 1992), pp. 139-206.

[6] L. F. DiMauro and P. Agostini, Adv. At. Mol. Phys. 35, 79 (1995).

[7] J. J. Macklin, J. D. Kmetec, and C. L. Gordon III, Phys. Rev. Lett. 70, 766 (1993)

[8] A. L'Huillier and P. Balcou, Phys. Rev. Lett. 70, 774 (1993).

[9] M. Lewenstein, P. Balcou, M. Yu. Ivanov, A. L'Huillier, and P. B. Corkum, Phys. Rev. A 49, 2117 (1994).

[10] W. Becker, S. Long, and J. K. McIver, Phys. Rev. A 50, 1540 (1994).

[11] K. Kendo, T. Tamida, Y. Nabekawa, and S. Watanabe, Phys. Rev. A 29, 3881 (1994).

[12] M. D. Perry and J. K. Crane, Phys. Rev. A 48, R4051 (1993).

[13] S. Long, W. Becker, and J. K. McIver, Phys. Rev. A 52, 2262 (1995)

[14] M. Protopapas, A. Sanpera, P. L. Knight, and K. Burnett, Phys. Rev. A 52, R2527 (1995).

[15] M. Bao and A. F. Starace (unpublished).

[16] N. L. Manakov and L. P. Rapoport, Zh. Éksp. Teor. Fiz. 69, 842 (1975) [Sov. Phys. JETP 42, 430 (1976)].
[17] I. J. Berson, J. Phys. B 8, 3078 (1975).

[18] Yu. N. Demkov and V. N. Ostrovskii, Zero-Range Potentials and Their Applications in Atomic Physics (Plenum, New York, 1988).

[19] M. Bao and A. F. Starace, Comput. Phys. 10(1), 89 (1996).

[20] B. Sundaram and P. W. Milonni, Phys. Rev. A 41, R6571 (1990).

[21] One may follow the steps given explicitly in Eqs. (3.9)-(4.2) of Ref. [10], using the vector potential defined in our Eq. (3).

[22] Cf. Eqs. (4.1) and (4.2) of Ref. [10]. Note that our $\mathbf{d}(\Omega)$ in Eq. (12) differs from Eq. (4.1) of Ref. [10] (in the limit $E_{S} \rightarrow 0$ ) by a factor $-i / 2$.

[23] H. B. van Linden van den Heuvel and H. G. Muller, in Multiphoton Processes, edited by S. J. Smith and P. L. Knight (Cambridge Univ. Press, Cambridge, 1988).

[24] T. F. Gallagher, Phys. Rev. Lett. 61, 2304 (1988).

[25] J. L. Krause, K. J. Schafer, and K. C. Kulander, Phys. Rev. Lett. 68, 3535 (1992); K. J. Schafer, B. Yang, L. F. DiMauro, and K. C. Kulander, ibid. 70, 1599 (1993).

[26] P. B. Corkum, Phys. Rev. Lett. 71, 1994 (1993).

[27] Q. Wang and A. F. Starace, Phys. Rev. A 48, R1741 (1993); 51, 1260 (1995).

[28] L. V. Keldysh, Zh. Éksp. Teor. Fiz. 47, 1945 (1964) [Sov. Phys. JETP 25, 1307 (1965)]. See also Refs. [6] and [14].

[29] J. H. Eberly, Q. Su, and J. Javanainen, Phys. Rev. Lett. 62, 881 (1989); J. Opt. Soc. Am. B 61289 (1989).

[30] G. Bandarage, A. Maquet, and J. Cooper, Phys. Rev. A 41, R1744 (1990).

[31] W. Becker, S. Long, and J. K. McIver, Phys. Rev. A 46, R5334 (1992); see also Ref. [10]. 